\title{
Le joueur bavard. Vers une typologie du dialogue dans le jeu vidéo
}

\author{
Jérôme-Olivier Allard \\ Université de Montréal
}

Les jeux de rôle pour ordinateur (Computer role-playing games, CRPG) contemporains sont les héritiers de fictions textuelles interactives, dont Zork (Infocom, 1980), du jeu sur table Dungeons \& Dragons et des Livres dont vous êtes le héros. Ces jeux fortement narratifs, souvent ancrés dans des univers inspirés de la high fantasy tolkienienne et de la science-fiction, comptent pour la plupart ces éléments de jouabilité : l'exploration d'un monde fictionnel plus ou moins vaste; les combats (en temps réel ou différé); l'amélioration des aptitudes 
du personnage-joueur par le biais d'un système de points d'expérience et de gain de niveaux; la gestion d'un équipement; la poursuite de quêtes diverses; et l'interaction avec une pléthore de personnages-non-joueurs. Si plusieurs jeux mettent en scène des conversations entre le personnage-joueur et des personnages-non-joueurs, sous la forme de cinématiques ou de séquences intégrées à l'action du jeu - pensons aux discussions qui meublent les déplacements plus monotones dans Red Dead Redemption (Rockstar, 2010) - où le joueur ne contrôle pas ce qui est dit, les jeux de rôle, comme certains jeux d'aventure, ont souvent la particularité de permettre au joueur de choisir ce que son personnage dit.

Dans un article consacré au dialogue dans Final Fantasy VII, Greg Smith (2002) remarque que si les études cinématographiques ont récemment tenté de dresser un portrait des fonctions spécifiques du dialogue dans ce média, les études vidéoludiques, encore récentes, tardent toujours à le faire. De fait, si certains auteurs ont consacré des ouvrages et des articles au dialogue dans la littérature (Coltier, 1990; Durrer, 1999; Denoyelle, 2010) et au cinéma (Field, 1990; Kozloff, 2000; Vassé, 2003), peu de chercheurs se sont intéressés au dialogue dans un corpus vidéoludique. Il appert donc pertinent, dans le contexte de ce dossier consacré aux résonances médiatiques, de tracer l'ébauche d'une typologie du dialogue dans les CRPG contemporains, où le joueur occupe un rôle singulier dans l'organisation du récit.

Dragon Age II (BioWare, 2011), jeu de rôle à la troisième personne inspiré du genre de la fantasy, m'est apparu particulièrement éclairant dans le cadre d'une telle réflexion, non seulement en raison de la qualité de l'écriture des scènes 
dialoguées qui y sont présentées, mais aussi à cause du rôle important de ces dernières dans la construction du récit. Le jeu, qui explore notamment les thèmes de la loyauté, de la bigoterie, du racisme et des idéaux révolutionnaires, se déroule sur le continent de Thedas, qui est déchiré par des tensions grandissantes entre les mages et les templiers, une faction religieuse censée protéger les citoyens des dangers de la magie. Le joueur tient le rôle de Hawke, un homme (ou une femme ${ }^{1}$ ) qui, après avoir été chassé de chez lui par les événements relatés dans Dragon Age: Origins (BioWare, 2009), doit reconstruire sa vie dans la ville de Kirkwall. Pendant les six ans que dure l'histoire du jeu, Hawke devient l'un des personnages les plus importants de Thedas et occupe une place de premier plan dans les événements qui mènent au déclenchement de la guerre entre les mages et les templiers. Au cours de ses premières années à Kirkwall, Hawke rencontre sept compagnons - Aveline, Varric, Anders, Isabella, Fenris, Merrill et Sebastian - qui, en plus de son frère Carver ${ }^{2}$ ou de sa sœur Bethany, suivent le personnage-joueur dans sa quête de gloire et de pouvoir. Lorsqu'il part à l'aventure, le joueur peut à sa guise choisir jusqu'à trois de ces alliés pour accompagner Hawke, et optimiser le groupe en fonction des aptitudes de chaque personnage.

\footnotetext{
1 À l'instar de moult jeux de rôle, Dragon Age II permet au joueur de tenir le rôle d'un homme ou d'une femme, guerrier, mage ou voleur. Afin d'éviter d'alourdir le texte et puisque j'ai joué un homme lors de mon séjour dans Thedas, j'emploierai le masculin pour décrire Hawke.

2 Un seul des jumeaux cadets de Hawke survit aux événements se déroulant dans le prologue du jeu. Si le personnage-joueur est un mage, Bethany meurt; s'il est un guerrier ou un voleur, Carver meurt. Le Hawke que j'ai incarné étant un mage, c'est donc Carver qui l'a accompagné dans ses aventures.
} 
$\mathrm{Si}$, pour Francis Berthelot, «les héros de romans sont surtout de grands bavards » (2003, p. 1), Hawke, à l'instar de ses cousins littéraires, passe de longs moments à discuter avec ses compagnons et avec les différents personnages qu'il rencontre au fil de son parcours. Dans le cadre de cet article, qui participe d'une réflexion plus large sur les recoupements entre littérature et jeu vidéo, je défendrai la thèse selon laquelle le dialogue dans Dragon Age II, qui occupe plusieurs fonctions héritées d'autres médias ou propres aux œuvres vidéoludiques, participe à la construction de l'univers fictionnel et enrichit l'expérience du joueur. Pour ce faire, je me propose d'emblée de définir brièvement les notions de jeu et de récit vidéoludique, avant de préciser les formes qu'adopte le dialogue dans Dragon Age II, de même que les fonctions qu'il remplit.

\section{Le récit vidéoludique}

À l'instar de plusieurs chercheurs, je considère que le débat fondateur des études vidéoludiques ${ }^{3}$, s'il a prouvé la pertinence

\footnotetext{
3 À l'orée du XXIe siècle, les études vidéoludiques naissantes sont le site d'un débat épistémologique opposant deux postures théoriques en apparence irréconciliables: celle des ludologues et celle des narratologues (Jenkins, 2004). Bien que cette querelle, souvent polémique et entretenue de manière un peu artificielle, soit fondée principalement sur des malentendus (Frasca, 2003; Aarseth, 2012) et sur des imprécisions définitionnelles (Ryan, 2006), notamment en regard de la notion de récit, il demeure pertinent, dans le cadre d'une réflexion sur les dialogues dans les jeux vidéo de rôle, de rappeler très brièvement les deux positions. Les ludologues, avec à leur tête notamment Gonsalo Frasca (1999) et Jesper Juul (1999), soutiennent que le jeu vidéo n'est pas un média intrinsèquement narratif; Markku Eskelinen (2001) va même jusqu'à écrire que l'histoire dans certains jeux n'est qu'un emballage superflu et que l'étudier est une perte de temps. Les narratologues défendent quant à eux le point de vue contraire : (tous) les jeux sont des récits (Murray, 2004) et les étudier à l'aide des outils issus des études littéraires et de la
} 
de développer des outils méthodologiques propres à l'étude des jeux vidéo, ne mérite pas d'être rouvert aujourd'hui. Plusieurs jeux vidéo récents, pensons entre autres à Gone Home (Fullbright, 2014), constituent des objets narratifs très riches, abordant des thèmes complexes et présentant des histoires nuancées qui méritent qu'on s'y intéresse. En guise de prémisse à une réflexion sur le dialogue dans les jeux vidéo, il demeure cependant important de rappeler les particularités du récit vidéoludique. Dans son ouvrage Half-Real, Jesper Juul, à la suite notamment de Roger Caillois, de Johan Huizinga et de Bernard Suits, définit le jeu comme

a rule-based system with a variable and quantifiable outcome, where different outcomes are assigned different values, the player exerts effort in order to influence the outcome, the player feels emotionally attached to the outcome, and the consequences of the activity are negotiable (2005, p. 36).

Pour Juul, le jeu vidéo est un objet duel, qui possède des règles réelles, mais qui se déroule dans un univers fictionnel. Selon l'auteur, c'est l'interaction entre les règles et la fiction qui est cruciale dans les jeux vidéo et qui les singularise par rapport aux autres types de jeux traditionnels. Juul (2002) distingue les jeux d'émergence (qui contiennent un nombre restreint de règles simples qui peuvent être combinées afin de former des variations intéressantes) et les jeux de progression (qui

narratologie cinématographique est pertinent. Marie-Laure Ryan adopte, notamment dans Avatars of Story (2006), une position plus nuancée. Pour l'auteure, certains jeux ont un clair dessein narratif; d'autres en ont moins et d'autres n'en ont tout simplement pas. Ryan propose par ailleurs, à la suite de David Herman, de penser les jeux comme des «narratively organized systems for playing » (p. 197), ce qui n'est pas sans rappeler la notion de « integrated crossmedia package » (2012) de Espen Aarseth, pour qui les jeux sont des ensembles pouvant émuler différents médias, que ce soit le roman, le film ou la bande dessinée, avec qui ils entrent en résonance. 
consistent en une série de défis qui s'enchaînent). La plupart des jeux vidéo s'inscrivent à un endroit ou à un autre du continuum dont l'émergence et la progression pourraient constituer les pôles ${ }^{4}$.

Dans un même ordre d'idées, Katie Salen et Eric Zimmerman (2004), à la suite de Marc LeBlanc, identifient deux types de récits vidéoludiques qui convoquent les catégories de jeu proposées par Juul : le récit enchâssé, qui existe avant que le joueur n'intervienne dans le jeu (pensons à Peach qui est constamment kidnappée par Bowser et que Mario doit sauver) et le récit émergent, c'est-à-dire qui est généré spontanément dans l'espace du jeu et qui est le résultat de l'interaction du joueur avec les règles. Par exemple, le Dovahkiin, protagoniste du jeu The Elder Scrolls V: Skyrim (Bethesda, 2011), après avoir classé des livres dans sa bibliothèque, vole tous les gâteaux d'un hameau, tue deux dragons et part batifoler dans la plaine pour chasser des papillons. Plusieurs jeux de rôle, dont Skyrim et Dragon Age II, présentent à mon sens - et ce, autant sur le plan de la jouabilité que sur celui de la narration - une structure hybride, plus ou moins à mi-chemin entre émergence et progression. De fait, les jeux de rôle accordent au joueur une place importante dans l'organisation du récit vidéoludique : en faisant des choix et en posant des actions dans le monde virtuel, le joueur a un impact sur l'évolution de l'histoire. On parle donc souvent des jeux de rôle en termes de récits non linéaires ou à embranchements. Ceci n'est pas sans rappeler le cybertexte

\footnotetext{
4 À titre d'exemple, on pourrait qualifier les jeux d'aventure en pointer-cliquer comme Day of the Tentacle (LucasArts, 1993) de jeux de progression avec très peu d'émergence, et les jeux dits sandbox, dont Minecraft (Mojang, 2009) est sûrement l'un des exemples le plus probants, comme des jeux d'émergence avec peu de progression.
} 
défini par Espen Aarseth (1997), dans lequel le lecteur est constamment au fait des stratégies et des chemins qui lui demeurent inaccessibles à cause des choix qu'il doit abandonner.

\section{Formes du dialogue}

Le jeu vidéo est un objet d'étude récent. Par conséquent, il importe, lorsqu'on cherche à analyser sa dimension narrative, de tenir compte des apports théoriques d'autres disciplines plus anciennes, comme les études littéraires et cinématographiques, en prenant toutefois grand soin de valider la pertinence de chaque emprunt méthodologique. En s'appuyant sur les écrits de Michel Chion portant sur le son au cinéma, Claire Vassé distingue trois types de dialogue en fonction de la localisation dans l'image de la source de l'énonciation: le dialogue $i n$, dont on voit l'énonciateur à l'écran; le dialogue hors champ, dont on ne voit pas l'énonciateur, qui est néanmoins situé dans le même temps et dans un espace adjacent à l'action qui est montrée à l'écran; et le dialogue off, dont l'énonciateur est invisible et n'appartient ni au même temps ni au même lieu que l'action (2003, p. 27-28).

À la lumière des observations de Vassé, il est possible de distinguer, dans Dragon Age II, trois formes de dialogues: les cinématiques, les séquences interactives et le party banter, c'est-à-dire les échanges apparemment impromptus entre les compagnons de Hawke et dont le protagoniste est, la plupart du temps, exclu. Ces derniers dialogues, qui se déroulent pendant les séquences d'exploration ou de combat, sont activés de manière aléatoire ou scriptée (en fonction des compagnons 
présents, après une quête particulière, lors de l'arrivée dans une zone précise de Kirkwall, etc.) Les trois formes du dialogue observées dans le jeu de BioWare peuvent chevaucher les types proposés par Vassé. Il appert que les cinématiques et les séquences interactives présentent le plus souvent des dialogues in (on voit Hawke et son interlocuteur à l'écran), et le party banter, des dialogues hors champ (les personnages se trouvent souvent derrière Hawke, à la limite ou en dehors du cadre de la « caméra » contrôlée par le joueur). Dragon Age II ne contient à ma connaissance aucune occurrence de dialogue off, contrairement à Dragon Age: Inquisition (BioWare, 2014), où le protagoniste entend les échos oniriques d'une conversation à laquelle il a participé quelque temps avant.

Dragon Age II marque une rupture avec le premier opus de la série et avec la tradition des jeux de rôle en perspective isométrique de la fin des années 1990, dont Planescape: Torment (Black Isle Studios, 1999). Le joueur ne doit plus choisir parmi plusieurs lignes de dialogue qui représentent intégralement ce que le personnage dit, mais il doit manipuler une roue dialogique (dialogue wheel), où il a le choix entre quelques courtes paraphrases qui synthétisent les paroles du personnage. Ce nouveau paradigme adopté du premier Mass Effect (BioWare, 2008) témoigne peut-être du souci des producteurs de rendre le jeu plus accessible aux joueurs sur console. Il permet néanmoins à Dragon Age II d'instaurer un système de seize icônes indiquant au joueur le ton ou l'effet possible de la phrase que son personnage s'apprête à dire. Hawke peut tour à tour être diplomate, comique ou agressif; il peut notamment commencer une relation amoureuse, soudoyer un personnage ou interrompre le dialogue pour engager le combat. Il peut aussi, grâce à l'icône représentant un point 
d'interrogation, ouvrir un sous-menu lui permettant d'en apprendre davantage sur le personnage ou la situation qui est au cœur de la conversation. La roue dialogique avec icônes telle qu'elle est implémentée dans Dragon Age II permet en somme au joueur d'évaluer avec plus de précision la portée des paroles de son personnage: il lui est donc possible, par exemple, d'éviter de flirter accidentellement avec un compagnon, alors qu'il tentait tout simplement de se montrer empathique.

\section{Fonctions du dialogue}

Si Richard Rabil (2011) juge la qualité des dialogues vidéoludiques en fonction de quatre critères (utilité, crédibilité, originalité et interactivité), peu de chercheurs en études du jeu vidéo ont tenté d'établir une typologie de leurs fonctions; c'est pourquoi la réflexion développée ici s'appuiera sur des travaux issus des études littéraires et cinématographiques. Chercheuse en littérature, Sylvie Durrer (1999, p.117-121) dégage des écrits d'André Malraux quatre fonctions du dialogue dans le roman: la fonction d'exposition (le dialogue vise à faire connaître au lecteur des événements narratifs); la fonction de caractérisation (le dialogue renseigne sur les personnages, leur personnalité, leurs motivations, etc.); la fonction d'action (le dialogue fait avancer le récit); la fonction de transition (le dialogue sert de lien pour introduire la description d'un personnage ou d'un nouvel élément narratif). Corinne Denoyelle (2010, p. 2) fait, quant à elle, ressortir des travaux de Danielle Coltier quatre fonctions narratives des paroles de personnages romanesques: la fonction d'attestation (les paroles servent à attester de la justesse des propos du narrateur); la fonction de cohésion (les paroles permettent de 
justifier l'ordre de la narration); la fonction dramatique (les paroles deviennent des éléments importants de la diégèse et font progresser le récit); la fonction informative (les paroles donnent au lecteur de l'information sur les personnages et sur l'univers fictionnel).

Sarah Kozloff (2000, p. 33-51) soutient pour sa part que le dialogue occupe neuf fonctions au cinéma, dont six sont selon elles fondamentales : la création et l'ancrage de la diégèse et des personnages; la communication de la causalité narrative; la promulgation d'événements narratifs; la révélation des personnages; l'adhésion au code du réalisme (le dialogue est souvent une représentation d'une conversation ordinaire) et le contrôle des émotions du spectateur (le dialogue guide son interprétation). Syd Field (1990, p.44) estime enfin que " [l]e dialogue [au cinéma] doit communiquer des faits et des informations au public; il doit faire avancer l'action; il révèle la personnalité, les conflits et l'état émotif des personnages. Le dialogue découle du personnage. » Mais qu'en est-il dans le cas des jeux vidéo?

Ainsi qu'on peut le constater dans le tableau récapitulatif présenté ci-dessous, plusieurs fonctions du dialogue mises au jour par les chercheurs cités semblent se recouper et se compléter, et ce, indépendamment du média décrit. Mes réflexions (empiriques et toujours en chantier) m'amènent à postuler que les CRPG partagent avec les médias littéraire et cinématographique trois fonctions: narrative, diégétique et émotionnelle. Le jeu vidéo étant fondé sur l'action du joueur à l'intérieur d'un environnement immersif, je postule qui plus est l'existence d'une fonction immersive du dialogue, qui serait propre au média vidéoludique sans être tout à fait étrangère à 
la fonction d'adhésion au code du réalisme proposée par Kozloff. Par une brève analyse de Dragon Age II, j'entends ici justifier par des exemples concrets la typologie du dialogue dans les jeux de rôle que je propose.

Fonctions du dialogue

\begin{tabular}{|c|c|c|c|c|}
\hline $\begin{array}{l}\text { Durrer } \\
\text { (roman) }\end{array}$ & $\begin{array}{l}\text { Coltier } \\
\text { (roman) }\end{array}$ & $\begin{array}{l}\text { Kozloff } \\
\text { (cinéma) }\end{array}$ & Field (cinéma) & Jeu vidéo \\
\hline Exposition & \multirow[b]{2}{*}{ Informative } & $\begin{array}{l}\text { Ancrage de la } \\
\text { diégèse }\end{array}$ & $\begin{array}{c}\text { Communiquer } \\
\text { des informations }\end{array}$ & \multirow[b]{2}{*}{ Diégétique } \\
\hline Caractérisation & & $\begin{array}{l}\text { Révélation des } \\
\text { personnages }\end{array}$ & $\begin{array}{c}\text { Révéler la } \\
\text { personnalité des } \\
\text { personnages }\end{array}$ & \\
\hline Action & Dramatique & $\begin{array}{c}\text { Promulgation } \\
\text { d'événements } \\
\text { narratifs }\end{array}$ & $\begin{array}{l}\text { Faire avancer } \\
\text { l'action }\end{array}$ & \multirow{2}{*}{ Narrative } \\
\hline \multirow[t]{4}{*}{ Transition } & Cohésion & $\begin{array}{l}\text { Causalité } \\
\text { narrative }\end{array}$ & & \\
\hline & Attestation & $\begin{array}{l}\text { Adhésion au } \\
\text { code du } \\
\text { réalisme }\end{array}$ & & \\
\hline & & & & Immersive \\
\hline & & $\begin{array}{c}\text { Contrôle des } \\
\text { émotions du } \\
\text { spectateur }\end{array}$ & & Émotionnelle \\
\hline
\end{tabular}

\section{Fonction narrative}

Dans le jeu de BioWare, le dialogue est l'un des principaux moteurs du récit, dont il assure aussi la cohésion. Au cours de ses errances dans la ville de Kirkwall et dans les parcours sinueux de la Wounded Coast, Hawke rencontre différents personnages qui lui confient, lors de séquences dialoguées, diverses quêtes secondaires, de la classique fetch quest où on demande au personnage-joueur de retrouver puis de rapporter un objet, aux quêtes spéciales confiées par les compagnons du protagoniste lorsque celui-ci a gagné leur confiance. Une fois sa 
mission accomplie, le joueur doit de nouveau parler avec le personnage afin de compléter la quête et de recevoir sa récompense. C'est, de plus, lors de séquences dialogiques que le joueur prend les décisions importantes qui ont une répercussion ${ }^{5}$ sur l'histoire du jeu et sur ses différents personnages. Hawke est entre autres amené à se positionner à de nombreuses reprises par rapport au conflit qui oppose les mages aux templiers. Dans la dernière séquence du jeu, alors que la confrontation ultime entre les troupes du First Enchanter Orsino et de la Knight-Commander Meredith est imminente, le joueur doit d'ailleurs choisir une dernière fois, par le biais de la roue dialogique, qui il appuie. Ce choix final effectué par Hawke détermine à la fois le type d'ennemis qu'il doit affronter dans les dernières minutes du jeu et le contenu de l'épilogue de Dragon Age II, où le protagoniste peut être nommé vicomte de Kirkwall, s'il a appuyé les templiers, ou être forcé de s'exiler, s'il a appuyé les mages.

En fonction des choix que le joueur a effectués précédemment lors de séquences de dialogue, certaines possibilités s'offrent à lui, alors que d'autres lui sont retirées. À titre d'exemple, si Hawke a opté la plupart du temps pour des réponses diplomates, il peut parfois éviter le combat et trouver une solution pacifique à une situation envenimée. S'il s'est plutôt montré agressif dans ses réponses, il peut intimider un personnage et le forcer à lui révéler une information secrète. Dans son ouvrage séminal Cybertext, Aarseth décrit le lecteur d'un cybertexte comme un joueur à risque, qui se démène pour gagner le contrôle narratif $(1997$, p. 4). Il semble que le joueur

${ }^{5}$ Les choix réalisés par le joueur ont non seulement un impact sur la trame narrative de Dragon Age II, mais aussi, par le biais d'un système d'importation de l'état du monde (world state), sur celle de la suite, Dragon Age: Inquisition. 
d'un CRPG, qui doit choisir les phrases prononcées par son avatar, se transforme en quelque sorte en ce lecteur dont parle Aarseth, conscient des voies narratives qui s'ouvrent et se ferment au fur et à mesure qu'il progresse dans l'arborescence dialogique, chaque choix de réponse devenant en quelque sorte l'une des branches du récit non linéaire mis en place dans le jeu.

\section{Fonction diégétique}

Les jeux vidéo disposent de plusieurs interfaces pour raconter une histoire au joueur et lui faire découvrir l'univers fictionnel dans lequel il évolue. Dans les jeux de rôle, c'est souvent par le biais du texte que le joueur acquiert des informations sur le monde du jeu, que ce texte prenne la forme d'entrées encyclopédiques ou d'objets-livres intraludiques (Allard, 2015). Notons à cet égard que Dragon Age II compte environ trois cent cinquante entrées de codex et que Skyrim contient plus de quatre cents livres que le joueur peut découvrir, lire et collectionner dans le jeu. C'est néanmoins aussi à travers les dialogues entre le personnage-joueur et la pléthore de personnages-non-joueurs qui peuplent Kirkwall que les informations relatives à l'histoire et à l'univers du jeu de BioWare sont révélées au moment opportun. C'est de plus grâce aux conversations qu'il a avec ses compagnons que le joueur en vient à connaître leur personnalité, leur histoire et leurs motivations, informations auxquelles il ne peut accéder qu'en les écoutant parler.

À titre d'exemple, Hawke entretient avec son frère Carver

une relation conflictuelle teintée de rivalité. Le jeune homme a 
l'impression de vivre constamment dans l'ombre de son frère aîné. En témoigne notamment cet échange qu'il a avec Varric :

Carver : Don't look at me. / Varric: What's your issue now, little Hawke? / Carver : Don't call me... just don't, alright? You're just looking for fodder for your stories. / Varric : You think you're that interesting? / Carver: I have enough trouble being overshadowed as it is. I don't need to get caught under an imaginary me, too./ Varric: Don't you worry. I'm not in the business of lullabies or children's stories.

Le joueur, qui en apprend plus ici sur le sentiment d'infériorité de Carver, peut par la suite adapter sa façon d'entrer en relation avec le personnage : il lui est possible de décider de le mettre en valeur ou, au contraire, de miner la confiance en soi du jeune homme. En somme, dans Dragon Age II, le dialogue informe le joueur; il est une clé qui lui donne accès au savoir, qu'il soit relatif au monde de Thedas ou aux personnages qui le peuplent. À cet égard, mentionnons que, dans les opus de la saga de BioWare, certaines options de dialogue permettent au joueur de déverrouiller des entrées de codex qui le renseignent sur l'univers fictionnel mis en scène dans le jeu.

\section{Fonction immersive}

Dans les formes romanesque et filmique, l'insertion d'un dialogue contribue à créer un effet de réel qui consolide le pacte de lecture ou de spectature et renforce l'adhésion au code cinématographique du réalisme, phénomène qui est aussi selon moi présent dans les jeux vidéo. Ceux-ci offrent néanmoins au joueur une expérience unique en termes d'immersion dans l'œuvre, qui n'est pas permise par les médias non interactifs; c'est pourquoi je propose l'existence d'une fonction immersive 
spécifique. Si Vassé soutient qu'au cinéma, le spectateur est a priori exclu du dialogue, cet "échange intime [entre les personnages] qui a déjà eu lieu » (2003, p. 16), le joueur occupe quant à lui un rôle singulier dans les séquences dialoguées. À mon sens, non seulement ces dernières n'interrompent pas la jouabilité dans Dragon Age II, mais elles participent au maintien d'un sentiment d'immersion, en misant notamment sur la participation du joueur, de manière semblable aux cinématiques dont Rune Klevjer (2002) se fait le défenseur. De fait, puisqu'il doit régulièrement choisir quelle réponse donner et qu'il a conscience des options qu'il doit abandonner, le joueur en situation de dialogue déploie toujours l'effort ergodique dont parle Aarseth (1997).

S'inscrivant de manière très nette dans la tradition des jeux de rôle d'inspiration Dungeons \& Dragons, Dragon Age II permet d'entrée de jeu au joueur de tracer les traits grossiers de l'identité de son personnage en choisissant son sexe et sa classe (guerrier, mage ou voleur) et en personnalisant son apparence. C'est toutefois par le biais des dialogues que se dessine la personnalité de Hawke. Comme je l'ai évoqué plus tôt, la roue dialogique permet au joueur d'opter pour des réponses diplomates, comiques ou agressives. Le système informatique du jeu comptabilise chaque réponse donnée et détermine celle qui est choisie le plus souvent. C'est cette accumulation de choix répétés dans le temps qui confère à Hawke l'illusion d'une identité stable, qui serait révélée par le jeu ${ }^{6}$. En plus d'ouvrir et de fermer certaines voies narratives, la

6 Je paraphrase ici les propos de Judith Butler, qui décrit dans un autre contexte l'identité de genre comme étant performative : «Le genre, c'est la stylisation répétée des corps, une série d'actes répétés à l'intérieur d'un cadre régulateur des plus rigides, des actes qui se figent dans le temps de telle sorte 
personnalité du personnage-joueur a notamment une incidence sur les interjections qu'il lance automatiquement pendant les séquences d'action, lorsqu'il est blessé ou qu'il triomphe d'un ennemi. Un Hawke diplomate rallie ses alliés en poussant un «Follow my lead! » convaincu, alors qu'un Hawke blagueur laisse pour sa part tomber un «And we're under attack. What a surprise! » teinté de sarcasme.

À mon sens, le fait pour le joueur de voir se concrétiser ainsi les choix qu'il a faits pendant les séquences de dialogue renforce le sentiment d'immersion grâce à l'identification à l'avatar. Smith (2002) remarque d'ailleurs que, lorsque le joueur choisit de faire agir son personnage de telle ou telle manière, il devient complice de ses actions, il adopte son comportement. En d'autres mots, le joueur devient son personnage. Notons à cet égard que, puisque Dragon Age II est un jeu de rôle, le plaisir du joueur semble se situer d'abord dans la mimicry, pour reprendre l'expression employée par Caillois (1992), c'est-à-dire dans le faire-semblant. Or, Dominic Arsenault soutient que,

[c]ontrairement à ce que le vocable pris en lui-même pourrait suggérer, le jeu de rôles informatique [...] ne repose pas sur le principe de «jouer un rôle» (au sens fort de la mimicry de Caillois: l'imitation et le faire-semblant caractéristique, notamment, du jeu du comédien). Le jeu de rôles vidéoludique [...] n'a que bien peu à voir avec l'incarnation mimétique d'un personnage que l'on retrouve dans un jeu de rôles sur table ou grandeur nature. (2011, p. 159; l'auteur souligne)

À l'instar d'Arsenault, je crois qu'il ne faut pas conclure de manière trop hâtive à une équivalence entre CRPG et jeux de

qu'ils finissent par produire l'apparence de la substance, un genre naturel de l'être. » (2005, p. 109-110) 
rôle sur table. J'ai néanmoins l'intuition que certains joueurs de jeux vidéo de rôle - pour qui la construction du personnagejoueur revêt une importance significative - en viennent à s'identifier avec une intensité particulière à leur avatar (Hawke, dans le cas qui nous intéresse), oscillant dans leur expérience vidéoludique entre transe et dépendance, pour reprendre la terminologie de Ryan, qui distingue quatre degrés d'absorption de l'acte de lecture ${ }^{7}$. Jacques Henriot écrit que, « [p] our jouer, il faut entrer dans le jeu. Pour entrer dans le jeu, il faut savoir que c'est un jeu. Il y a donc, de la part de celui qui se met à jouer, une compréhension préalable du sens du jeu. L'attitude ludique, comme toute attitude, se prend. Comme toute attitude, elle se comprend. » (1983, p. 83) C'est peut-être au final la disposition du joueur à se prêter au jeu et l'attitude ludique qu'il adopte qui fixent en quelque sorte son degré d'immersion dans l'œuvre, contribuant à moduler son attachement aux personnages-nonjoueurs auxquels il se lie sur un mode fantasmatique par l'entremise des dialogues.

\section{Fonction émotionnelle}

De manière analogue au système de réputation de Baldur's Gate (BioWare, 1998) et à celui d'approbation de Dragon Age: Origins, Dragon Age II tient compte des décisions du joueur afin de déterminer le type de relation que Hawke entretient avec ses

\footnotetext{
7 Pour Ryan, ces quatre degrés d'absorption sont la concentration (attention accordée aux textes non immersifs), l'implication imaginaire (posture partagée entre l'immersion dans un texte et sa contemplation esthétique), la transe (plaisir non réflexif du lecteur qui en vient à oublier ce qui est extérieur au texte) et la dépendance, pouvant prendre la forme d'une perte de contact avec la réalité (2001, p. 98).
} 
nombreux compagnons et d'évaluer la loyauté que ceux-ci éprouvent pour le protagoniste, loyauté qui peut prendre la forme d'une amitié sincère ou d'une rivalité respectueuse. La plupart des choix importants que le joueur doit faire, qu'on pense notamment à l'attitude de Hawke en regard du conflit qui oppose les mages et les templiers, sont perçus positivement ou négativement par les différents personnages et ont un effet sur leur relation avec le personnage-joueur.

Au fil de ses aventures, le joueur est amené à rendre visite à ses compagnons dans leur lieu de résidence afin de discuter de manière informelle. C'est lors de ces moments intimes, qui s'inscrivent en marge des séances d'action, que les personnages révèlent à Hawke leur passé, leurs préoccupations et leurs espoirs. En discutant avec les différents compagnons de son avatar, le joueur débloque aussi certaines quêtes qui, lorsqu'elles sont complétées, assurent à Hawke la loyauté de ses alliés. Ici, établir une relation favorable, qu'elle soit de nature amicale, amoureuse ou rivale, constitue en quelque sorte l'un des objectifs du jeu. En fonction des réponses qu'il choisit, le joueur peut en outre parvenir à changer la façon de voir les choses des personnages-non-joueurs. Déjà dans Baldur's Gate II (BioWare, 2000), il était possible, par le biais d'options de dialogue judicieusement choisies, de changer la personnalité de Viconia, une elfe drow maléfique. Dans Dragon Age II, à titre d'exemple, le joueur peut amener Fenris, un ancien esclave détestant la magie, à ne plus voir tous les mages comme des êtres mauvais et à considérer Hawke comme un ami.

À mon sens, c'est notamment du dialogue - où le joueur apprend à connaître, à aimer ou à détester les personnagesnon-joueurs - qu'émergent les émotions fictionnelles dont parle 
Bernard Perron (2006) à la suite d'Ed Tan, qui plongent le joueur dans l'univers du jeu et le rendent empathique envers son personnage. La psychologie cognitive nous apprend que le cerveau n'établit aucune différence entre un personnage fictionnel et un voisin qu'on croise de temps à autre. À cet égard, Ryan postule, à la suite de Richard Gerrig, que bien que nous sachions objectivement que les personnages fictionnels ne sont que des constructions linguistiques, cela ne nous empêche pas de les considérer comme s'ils étaient des humains en chair et en os (2001, p. 94). Ce n'est pas, en effet, parce qu'ils sont une accumulation de 0 et de 1 que les personnages de Dragon Age II ne méritent pas qu'on prenne la peine de leur parler. Le dialogue contribue ainsi à améliorer le jeu par son aptitude à faire vivre au joueur une expérience émotionnelle riche, incluant notamment des affects relatifs à l'attachement.

\section{Conclusion}

En somme, j'ai voulu proposer, dans cet article, une typologie des formes et des fonctions du dialogue dans les jeux vidéo de rôle, à la lumière des travaux d'un certain nombre de chercheurs qui ont effectué un travail similaire à partir d'un corpus littéraire ou cinématographique. J'ai ainsi postulé l'existence de trois fonctions (narrative, diégétique, émotionnelle), qui seraient communes à plusieurs médias, et d'une quatrième (immersive), qui serait propre au média interactif qu'est le jeu vidéo. À travers une analyse du dialogue dans Dragon Age II, il a par ailleurs été possible de montrer que celui-ci participe à la construction de l'univers fictionnel et enrichit l'expérience du joueur, confirmant ainsi l'hypothèse de travail proposée en introduction. Ma réflexion est toujours en 
chantier : il serait pertinent, voire nécessaire, de compléter cet essai typologique par l'analyse d'un corpus plus étendu; pensons notamment à la série The Elder Scrolls ou à des titres issus de la nouvelle vague de jeux de rôle en perspective isométrique. En terminant, s'il a été question ici de dialogues ayant été soigneusement scénarisés et organisés suivant une structure pour l'essentiel en progression, il faudrait tenir compte, dans l'élaboration d'une typologie plus inclusive du dialogue dans le jeu vidéo, des particularités du dialogue en langage naturel (associé à une structure dialogique émergente), tel qu'il se décline notamment dans le jeu Façade (Procedural Arts, 2005). 


\section{Bibliographie}

Aarseth, Espen. (1997), Cybertext. Perspectives on Ergodic Literature, Baltimore, Johns Hopkins University Press.

-. (2012), "A Narrative Theory of Games», Foundations of Digital Games, New York, ACM, p.129-133, <http://dl.acm.org/citation.cfm?id=2282365>.

ALLARD, Jérôme-Olivier. (2015), « Joue-moi une histoire. Objetlivre intraludique et ouverture du récit dans le jeu vidéo ", dans Fanny BARNABÉ et Björn-Olav Dozo (dir.), Livre et jeu vidéo, Liège, Bebooks, p. 28-49.

ARSENAULT, Dominic. (2011), «Des typologies mécaniques à l'expérience esthétique. Fonctions et mutations du genre dans le jeu vidéo », thèse de doctorat, Université de Montréal.

Berthelot, Francis. (2001), Parole et dialogues dans le roman, Paris, Nathan.

BioWARE. (2011), Dragon Age II, Edmonton, Electronic Arts.

ButLer, Judith. (2005), Trouble dans le genre. Pour un féminisme de la subversion, Paris, La Découverte.

CaIllois, Roger. (1992 [1958]), Les Jeux et les hommes, Paris, Gallimard.

Coltier, Danielle. (1990), «Dialogues de romans», Pratiques, no 65 (mars).

Denoyelle, Corinne. (2010), «La fonction dramatique du dialogue dans les romans médiévaux», Cahiers de narratologie, no 19 (décembre),

<http://narratologie.revues.org/pdf/6219>. 
DURRER, Sylvie. (1994), Le Dialogue romanesque. Style et écriture, Genève, Droz.

-. (1999), Le Dialogue dans le roman, Paris, Nathan.

EsKelinen, Markku. (2001), «The Gaming Situation», Game Studies, vol. 1, no 1 , <http://gamestudies.org/0101/eskelinen>.

FIELD, Syd. (1990), Scénario : la rédaction d'un scénario, de l'idée originale à la version finale, Montréal, Merlin.

HENRIOT, Jacques. (1983 [1969]), Le Jeu, Paris, Synonyme.

Jenkins, Henry. (2004), «Game Design as Narrative Architecture », electronic book review, $<$ http://www.electronicbookreview.com/thread/firstperson /lazzi-fair>.

JuUL, Jesper. (1999), "A Clash between Game and Narrative », $<$ http://www.jesperjuul.net/thesis $>$.

—. (2002), «The Open and the Closed: Games of Emergence and Games of Progression », Computer Games and Digital Cultures Conference Proceedings, Tampere, Tampere University Press, p. 323-329.

-. (2005), Half-Real. Video Games between Real Rules and Fictional Words, Cambridge, MIT Press.

Klevjer, Rune. (2002), "In Defense of Cutscenes », Computer Games and Digital Cultures Conference Proceedings, Tampere, Tampere University Press, p. 191-202.

Kozloff, Sarah. (2000), Overhearing Film Dialogue, Berkeley, University of California Press. 
MurRaY, Janet. (2004), "From Game-Story to Cyberdrama », First Person: New Media as Story, Performance, and Game, Cambridge, MIT Press, p. 2-12.

PERron, Bernard. (2006), "Jeu vidéo et émotions», dans Sébastien Genvo (dir.), Le game design de jeux vidéo. Approches de l'expression vidéoludique, Paris, L'Harmattan, p. 347-366.

RABIL, Richard. (2011), "Dedicated to Game Dialogue», Gamesauce, $<$ http://gamesauce.org/news/2011/02/14/dedicated-togame-dialogue-by-richard-rabi>.

RYAN, Marie-Laure. (2001), Narrative as Virtual Reality: Immersion and Interactivity in Literature and Electronic Media, Baltimore, Johns Hopkins University Press.

-. (2006), Avatars of Story, Minneapolis, University of Minneapolis Press.

Salen, Katie et Eric Zimmerman. (2004), Rules of Play. Game Design Fundamentals, Cambridge, MIT Press.

Smith, Greg M. (2002), "Computer Games Have Words, Too: Dialogue Conventions in Final Fantasy VII», Game Studies, vol. 2, no 2, <http://gamestudies.org/0202/smith>.

VASSÉ, Claire. (2003), Le Dialogue, du texte écrit à la voix mise en scène, Paris, Cahiers du cinéma. 


\title{
Résumé
}

Cet article, qui s'inscrit dans une réflexion plus large sur les recoupements entre littérature et jeu vidéo, défend la thèse selon laquelle le dialogue dans Dragon Age II, qui occupe plusieurs fonctions héritées d'autres médias ou propres aux œuvres vidéoludiques, participe à la construction de l'univers fictionnel et enrichit l'expérience du joueur. Pour ce faire, la démonstration définit brièvement les notions de jeu et de récit vidéoludique, avant de préciser les formes qu'adopte le dialogue dans Dragon Age II de même que les fonctions qu'il remplit.

\begin{abstract}
This article, which is part of a wider reflection on the intersections between literature and videogames, argues that the dialogue in Dragon Age II, which has several functions inherited from other media or unique to videogames, contributes to the construction of the fictional world and enriches the gamer's experience. To this end, the article briefly defines the concepts of game and videogame narrative before specifying the forms and functions of the dialogue in Dragon Age II.
\end{abstract}

